\title{
Integration of the MEDITEC system in the Radiology Department of the University of the Free State
}

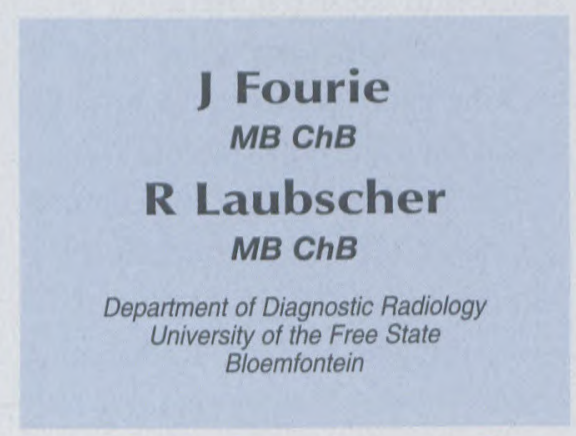

\section{History}

The MEDITEC system is a hospital information system (HIS) comprising various modules.

It was first incorporated into all three of the provincial hospitals in Bloemfontein in 1996. At first only the laboratory module was installed to process blood results faster. The final aim was to move to a more assessible and paperless system. Due to the success of the system it was decided to enlarge it to incorporate other modules..

The departmental module was incorporated at the beginning of 2000. The aim of the departmental module was not only to incorporate a paperless system, but also to give doctors permanent and immediate access to results obtained from special exam- inations done and previous patient data.

\section{Implementation}

The departmental module of the MEDITEC system consists of an empty shell that gives the person designing the system a free hand to customise the system to his/her department's individual needs. Doctors from every department were involved in designing the system.

In this article we will focus on how the system was customised to our department's individual needs and the advantages we get from using the system.

The three main components of the system are the application forms, the report forms and the retrieval of statistics from the system.

\section{Application forms}

The application form developed for the system was designed to be specific to the examination requested in that the questions on the form were appropriate to the examination done.

The application form was designed to give the full details automatically for the patient as well as the doctor requesting the examination. The advantage of this was that the requesting doctor could easily be reached. In a teaching hospital complex such as ours the doctors also vary from year to year.

The advantage of this type of request form is that some of the fields are mandatory and doctors are only asked questions pertinent to the type of investigation.

Mandatory fields play a role in ensuring patient safety, for example fields enquiring about metallic implants in requests for MRI investigations, or fields relating to known allergies to iodine in requests for examinations where contrast is likely to be administered.

These application forms can then be viewed on screen in the department or (as is the case in our department) they can be printed out automatically in the area where bookings for that examination are made.

Certain fields on the application form are examination-specific and are filled in by the radiographer who has done the procedure. These fields include the specific examinations done on the patient and the materials used. This enables the billing system to run smoothly.

A statistical field was designed specifically for use in our department and includes the code of the radiographer and/or student who did the examination, and the number and type of films passed and rejected.

\section{Report forms}

The reporting doctor has the advantage of having access, via the MEDITEC database, to all previous results such as haematology, cytology, previous radiological examinations and previous patient notes to assist 


\section{TECHNOLOGY TODAY}

him/her in making a diagnosis.

As a lot of patients have to go back to the referring clinics after the examination the films can now be given directly to the patient after the examination, and the referring doctor has access to the report on the system. The average time from the reporting doctor receiving the films to the report being available in a draft format on the system is about 20 minutes. This report is available not only to the referring doctor, but to all other doctors involved in caring for the patient.

The patient's information is accessed using a patient enquiry system. This system groups all the reports from all departments on one page and allows the doctor easy access to the relevant results.

The report can be viewed at Universitas and National hospitals (Pelonomi Hospital does not have the departmental module as yet) as soon as it is typed in our department.

All reports are signed digitally on the system after they have been checked by the reporting doctor. All changes to reports are monitored by the system for medical-legal reasons.

\section{Statistical use}

Information provided by the doctor as well as the attending radiographers is fed back into the system. These data can be recalled in any way depending on the statistics needed. For example:

- Ordering films in the department. The system can provide the exact amount and size of films used over any given time.

- As such a wide spectrum of information is fed back into the system, the data can also be used for compiling studies.

- Machine use can be evaluated by the number of examinations done per machine over a specific time.

- The work done by individual doctors can be monitored for individual statistics and work performance.

If the need arises for specific information, the application forms on the system are flexible enough to be changed easily so that additional data can be gathered.

\section{The future}

Although the system has already changed our department to a large extent there are other modules that can be added to the system. For example, the MEDITEC offers a PACS system for the storage of digital film and an internet module that makes patient information as well as X-ray films available to registered users.

Various other modules assist in booking patients and cost control for the hospital and departments.

The implementation and continuous use of the system was and still is without any problems. It is one of the few complete hospital information systems in the country that not only covers the whole spectrum of needs in a large hospital but because of its modular design also gives the system the ability to grow with the needs of the hospital. 